\title{
Clinically viable myocardial CCTA segmentation for measuring vessel-specific myocardial blood flow from dynamic PET/CCTA hybrid fusion
}

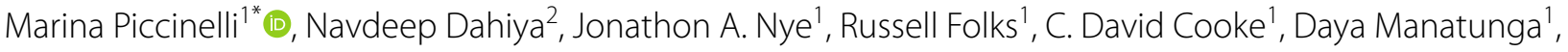 \\ Doyeon Hwang ${ }^{3}$, Jin Chul Paeng 4 , Sang-Geon Cho ${ }^{6}$, Joo Myung Lee ${ }^{5}$, Hee-Seung Bom ${ }^{6}$, Bon-Kwon Koo ${ }^{3}$, \\ Anthony Yezzi ${ }^{2}$ and Ernest V. Garcia'
}

\footnotetext{
${ }^{*}$ Correspondence:

mpiccin@emory.edu

${ }^{1}$ Department of Radiology

and Imaging Sciences,

Emory University School

of Medicine, 1364 Clifton Rd,

NE, Atlanta, GA 30322, USA

Full list of author information

is available at the end of the article
}

\begin{abstract}
Background: Positron emission tomography (PET)-derived LV MBF quantification is usually measured in standard anatomical vascular territories potentially averaging flow from normally perfused tissue with those from areas with abnormal flow supply. Previously we reported on an image-based tool to noninvasively measure absolute myocardial blood flow at locations just below individual epicardial vessel to help guide revascularization. The aim of this work is to determine the robustness of vesselspecific flow measurements $\left(\mathrm{MBF}^{\mathrm{vs}}\right.$ ) extracted from the fusion of dynamic PET (dPET) with coronary computed tomography angiography (CCTA) myocardial segmentations, using flow measured from the fusion with CCTA manual segmentation as the reference standard.
\end{abstract}

Methods: Forty-three patients' ${ }^{13} \mathrm{NH}_{3} \mathrm{dPET}$, CCTA image datasets were used to measure the agreement of the $\mathrm{MBF}^{\mathrm{vs}}$ profiles after the fusion of $\mathrm{dPET}$ data with three CCTA anatomical models: (1) a manual model, (2) a fully automated segmented model and (3) a corrected model, where major inaccuracies in the automated segmentation were briefly edited. Pairwise accuracy of the normality/abnormality agreement of flow values along differently extracted vessels was determined by comparing, on a pointby-point basis, each vessel's flow to corresponding vessels' normal limits using Dice coefficients (DC) as the metric.

Results: Of the 43 patients CCTA fully automated mask models, 27 patients' borders required manual correction before $\mathrm{APET} / \mathrm{CCTA}$ image fusion, but this editing process was brief (2-3 min) allowing a 100\% success rate of extracting MBF ${ }^{\mathrm{vs}}$ in clinically acceptable times. In total, 124 vessels were analyzed after dPET fusion with the manual and corrected CCTA mask models yielding 2225 stress and 2122 rest flow values. Fortyseven vessels were analyzed after fusion with the fully automatic masks producing 840 stress and 825 rest flow samples. All DC coefficients computed globally or by territory were $\geq 0.93$. No statistical differences were found in the normal/abnormal flow classifications between manual and corrected or manual and fully automated CCTA masks. 
Conclusion: Fully automated and manually corrected myocardial CCTA segmentation provides anatomical masks in clinically acceptable times for vessel-specific myocardial blood flow measurements using dynamic PET/CCTA image fusion which are not significantly different in flow accuracy and within clinically acceptable processing times compared to fully manually segmented CCTA myocardial masks.

Keywords: Multimodality image fusion, Absolute myocardial blood flow, Vesselspecific quantification, Cardiac PET, Coronary CTA

\section{Background}

Since the development and validation of the Fractional Flow Reserve (FFR) index (Bruyne and Sarma 2008) to invasively assess the hemodynamic significance of coronary lesions and guide revascularization (Bruyne et al. 2012), novel methodologies based on noninvasive imaging techniques have been investigated and proposed to stratify patients with known or suspected coronary artery disease (CAD) and plan the appropriate treatment. We previously proposed a novel technique based on a multimodality image fusion approach of dynamic positron emission tomography (dPET) and coronary CT angiography (CCTA) for the estimation of vessel-specific rest/stress $(\mathrm{r} / \mathrm{s})$ myocardial blood flow (MBF) along the three-dimensional (3D) trajectory of coronary vessels (Piccinelli et al. 2020). The rationale behind this methodology is the creation of an image-based tool to noninvasively estimate the $\mathrm{r} / \mathrm{s}$ absolute MBF and derived indexes, such as myocardial flow reserve (MFR) (Schindler et al. 2014) and relative flow reserve (RFR) (Lee et al. 2016), at precise locations underneath the perfusing vessel of interest and use it to guide treatment similarly to the invasive assessment of FFR during LV catheterization. The clinical implementation of this approach has potential for the improved localization of culprit lesions by increasing the mismatch between normal and abnormal flow measurements otherwise averaged when using results extracted from standard anatomical vascular territories.

Requirements for the clinical use of our approach include the multimodality image fusion of the APET data to CCTA-derived anatomy (i.e., the model of the biventricular myocardium including left (LV) and right (RV) ventricles) and our ability to efficiently extract such anatomical information without time-consuming manual processing. An automated technique for the segmentation of the biventricular epicardium (EPI), LV and RV profiles from CCTA images was recently published (Dahiya et al. 2019). A preliminary and thorough validation on the accuracy and robustness of measuring biventricular chambers size and myocardial mass was also recently reported (Piccinelli et al. 2021) with promising results on both aspects, particularly on its clinical feasibility. However, these studies did not determine the influence of the extracted anatomy on the fusion procedure and consequently on the accuracy of our vesselspecific flow $\left(\mathrm{MBF}^{\mathrm{vs}}\right)$ estimates.

The aim of this work was to determine the dependence of $\mathrm{MBF}^{\mathrm{vs}}$ measurements from the fusion of dPET with clinically viable CCTA myocardial segmentation, using as reference standard the $\mathrm{MBF}^{\mathrm{vs}}$ obtained from fusion of $\mathrm{dPET}$ with the manually delineated myocardium. Clinically viable segmentation was defined as fully automated segmentation of LV, RV and EPI borders followed by brief manual border 
editing in contrast to the two hours usually required for a complete manual border definition of the myocardium that could cover up to 250 CCTA slices.

The agreement between the differently obtained $\mathrm{MBF}^{\mathrm{vs}}$ was assessed using the Dice similarity coefficient (DC) (Dice 1945) and was determined in terms of classification of the resulting flow values in normal versus abnormal according to ranges for $\mathrm{MBF}^{\mathrm{vs}}$ developed with our methodology from a cohort of patients with low risk of CAD (Piccinelli et al. 2020).

\section{Methods}

Our overall approach to determine the role of the automated CCTA segmentation on MBF values was to measure the accuracy of $\mathrm{MBF}^{\mathrm{vs}}$ resulting from the fusion of dPET data with three differently extracted anatomical models. These models were as follows: (1) a totally manual $(\mathrm{M})$ model, which also represented our selected standard of reference, (2) a fully automated segmented (FA) model, obtained with our published technique (Dahiya et al. 2019; Piccinelli et al. 2021), and (3) a corrected (C) model, where major inaccuracies in the automated segmentation were identified during quality control assessment of the borders and briefly edited by an expert in cardiovascular anatomy. Inaccuracies to be manually corrected were visually identified as automated contours that markedly deviated from the myocardial surface or the chambers edges. The resulting three sets of $\mathrm{r} / \mathrm{s} \mathrm{MBF}^{\mathrm{vs}}$ were compared to previously developed ranges of flow normality along major vessels in the three vascular territories (Piccinelli et al. 2020) and their agreement was measured with a technique based on DC.

\section{Study population and imaging datasets}

Multimodality image datasets collected in the context of the multisite DEMYSTIFY study (AlBadri et al. 2020) (ClinicalTrails.gov registration number NCT04221594) were used for this investigation. Each dataset is comprised of static, gated and dynamic $\mathrm{r} / \mathrm{s}$ ${ }^{13} \mathrm{NH}_{3}$ PET, CCTA and invasive coronary angiograph (ICA). The first 43 cases included in the DEMYSTIFY database were selected for this study. These patient data were contributed by collaborators in S. Korea: Seoul National University Hospital (SNUH, 35 cases), Samsung Medical Center (SMC, 2 cases) and Chonnam National University Hospital (CNUH, 6 cases). The image acquisitions were performed after local IRB committee approval and consent forms were obtained from all subjects.

All centers used equivalent clinical protocols for both cardiac PET and CCTA imaging as previously reported (Piccinelli et al. 2020; AlBadri et al. 2020). Briefly, prior to the cardiac PET, patients were asked to fast overnight, abstain from caffeine-containing beverages and stop vasodilator medications (such as beta-blockers or calcium channel blockers) for $24 \mathrm{~h}$. The imaging protocol consisted of a low-dose CT scan for attenuation and scatter correction, followed by the injection of $370 \mathrm{MBq}$ bolus of ${ }^{13} \mathrm{NH}_{3}$ and the resting dynamic PET acquisition. Hyperemia was induced by a 3-min intravenous infusion of adenosine $(140 \mu \mathrm{g} / \mathrm{kg} / \mathrm{min})$ followed by a second dose of ${ }^{13} \mathrm{NH}_{3}$ and the stress PET acquisition. All images were acquired in list mode and binned according to the following temporal schemes: $12 \times 10 \mathrm{~s}, 6 \times 30 \mathrm{~s}, 2 \times 60 \mathrm{~s}, 1 \times 180 \mathrm{~s}$ for SNUH, $12 \times 5 \mathrm{~s}, 6 \times 10 \mathrm{~s}$, $3 \times 20 \mathrm{~s}, 6 \times 30 \mathrm{~s}$ for SMC and CNUH. Prospectively ECG-gated contrast-enhanced CT images were acquired following standard clinical guidelines after the injection of $60 \mathrm{~mL}$ 
of nonionic contrast agent at $4 \mathrm{~mL} / \mathrm{s}$. Sublingual nitroglycerine was administered to all patients to facilitate visualization of coronary vessels. The diastolic phase (usually located between 65 and $85 \%$ of the cardiac cycle) was selected for the extraction of the anatomy as it allowed a relative motion-free visualization of the major vessels and the myocardium. Coronary CTA images were acquired either before or after cardiac PET. Image acquisition was performed after local IRB committee approval and consent forms were obtained from all subjects. All images were saved in DICOM format, anonymized and securely transferred to the core laboratory at Emory University for subsequent processing.

\section{CCTA-derived anatomy extraction}

A requirement of our approach for the calculation of MBF along coronary 3D paths is the extraction of the anatomical information from the CCTA images, specifically binary masks identifying LV, RV and EPI, which are used for the 3D image fusion of dPET and CCTA images, and coronary centerlines to localize the myocardium underneath the vessels. The extraction of the anatomical masks is a particularly challenging task. If manually performed, it can require up to two hours work by a trained user. Fully automated segmentations may require a quality control assessment, but they guarantee time efficiency and clinical feasibility. The technical details of our automated segmentation algorithms are presented elsewhere, as well as an initial validation of their robustness when compared to manual segmentation used as the reference standard (Dahiya et al. 2019; Piccinelli et al. 2021).

After re-orientation of the transaxial CCTA images in the short-axis (SA) direction, an isotropic volume of $512 \times 512 \times 512$ pixels was created with the myocardium encompassing between 200 and 250 slices. A resampled version of this high-resolution dataset was created with a spacing $4 \times$ the native planar one and used as input to the automated algorithms. M masks were obtained by manual delineation of the myocardium and blood pools on the high-resolution volume by an expert in cardiac imaging using an in-house developed software for editing myocardial borders. FA masks were generated with our proposed automated algorithms (Dahiya et al. 2019) from the lowresolution version of the SA-oriented CT images. Finally, FA masks were reviewed and $\mathrm{C}$ masks obtained as the result of a limited manual correction step. The manual corrections had the final goal of ensuring the completion of the fusion procedure and specific guidelines were provided to perform such corrections. Since the image registration (as described in the next section) relied on a univocal identification of the ventricles and myocardium, specific features of the segmented heart morphology (e.g., a clear separation between epicardium and endocardium along the septum and at the apex) were targeted. Only additional major segmentation errors (e.g., heavy RV encroachment into the liver space) were manually corrected, but minor ones ignored. The aim of this investigation was not to measure the performance of our automated segmentation tools, but to determine whether low-resolved automated or semi-automated segmented masks obtained in time-efficient fashion could guide the fusion phase in the context of our proposed method for $\mathrm{MBF}^{\mathrm{vs}}$ quantification and lesion significance assessment. Efforts were made to minimize user interactions in order to truly assess the $\mathrm{MBF}^{\mathrm{vs}}$ calculation when guided by FA masks and mimic clinical environment settings where brief high-level user 
guidance in automated medical image processing tasks is common. LV, RV and EPI borders were reviewed slice-by-slice and minimally corrected where/if necessary. The time devoted to these corrections was recorded for each case. Finally, coronary centerlines were manually extracted with in-house developed image processing tools (VMTK 2021) from CCTA images. Figure 1 shows the 3D biventricular models with coronary centerlines obtained from the 3 sets of anatomical masks.

\section{PET/CCTA image fusion, flow model and vessel-specific MBF (MBF ${ }^{\text {vs }}$ ) calculation}

As previously described (Piccinelli et al. 2020), our methodology to calculate flow along vessel centerlines relies on the fusion of $\mathrm{dPET}$ and anatomy. Importantly, the dPET sequence was first corrected for inter-frame motion (Nye et al. 2021) and the resulting images were used for the remainder of the processing. The registration procedure consisted of the following steps (performed for rest and stress separately): (1) a summed PET image $\left(\mathrm{PET}_{\text {sum }}\right)$ was created from the dynamic frames of the second half of the acquisition interval; (2) the LVs segmented from the CCTA and $\mathrm{PET}_{\text {sum }}$ were rigidly registered; and (3) mutual information-based techniques were used to align $\mathrm{PET}_{\text {sum }}$ with the CCTA-derived biventricular mask using LV and RV structures from both modalities. More detailed information can be found in previous works (Faber et al. 2011; Piccinelli et al. 2018). The same transformations were finally applied to align the coronary centerlines to the PET space. Using the registered centerline as guidance, the myocardium subtended to each vessel was identified on the APET images and discretized in contiguous cubic volumetric elements with a longitudinal size of $4 \mathrm{~mm}$ that followed the 3D trajectories. The MBF quantification was performed using standard tracer kinetic modeling techniques. Time-varying tracer concentrations were extracted in the form of time activity curves (TAC) from specific regions of interests (ROIs), namely vascular territories and the arterial blood, and used as input data into a 2-tissue compartmental model (Hutchins et al. 1990) that returned r/s MBF values for each identified ROI. The TACs from the contiguous cubic ROIs representing the myocardium subtended to vessels were also fed to the model, thus generating $\mathrm{r} / \mathrm{s}$ flow profiles along the vessel $\left(\mathrm{MBF}^{\mathrm{vs}}\right)$. As the fusion procedure was performed with $\mathrm{M}, \mathrm{FA}$ and $\mathrm{C}$ anatomical masks, 3 sets of $\mathrm{r} / \mathrm{s}$ $\mathrm{MBF}^{\mathrm{vs}}$ curves were extracted for each analyzed vessel (respectively, $\mathrm{MBF}_{\mathrm{M}}^{\mathrm{vs}}, \mathrm{MBF}_{\mathrm{FA}}^{\mathrm{vs}}$ and $\mathrm{MBF}_{\mathrm{C}}^{\mathrm{vs}}$ ). Figure 2 shows the crucial processing steps for one of the analyzed cases: (A) the

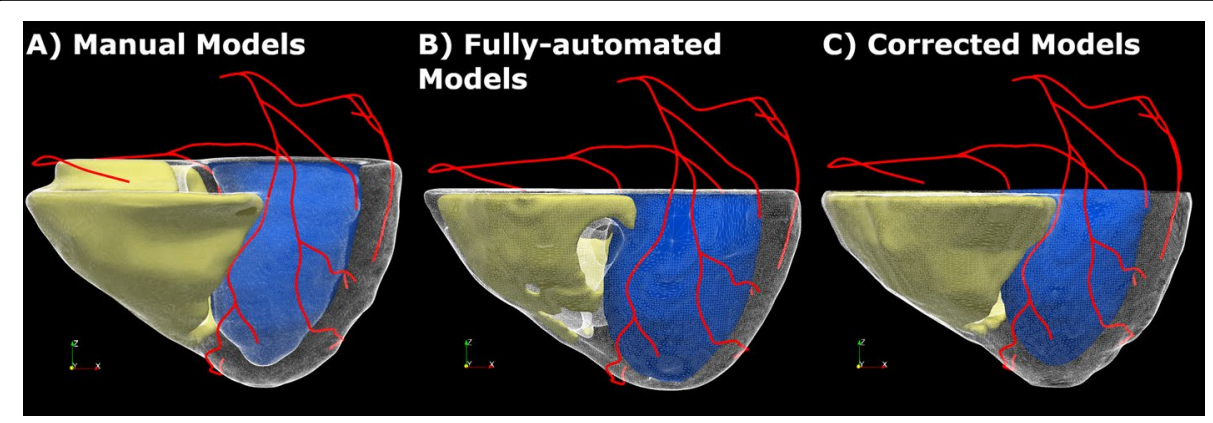

Fig. $13 D$ models reconstructed from the CCTA-derived anatomical binary masks of LV, RV and EPI obtained by manual delineation (A), by means of a fully automated segmentation algorithm $(\mathbf{B})$ and by manual correction of the automatically obtained masks (C). CCTA-derived coronaries centerlines also shown on all models. $L V$ left ventricle, $R V$ right ventricle, EPI biventricular epicardium 


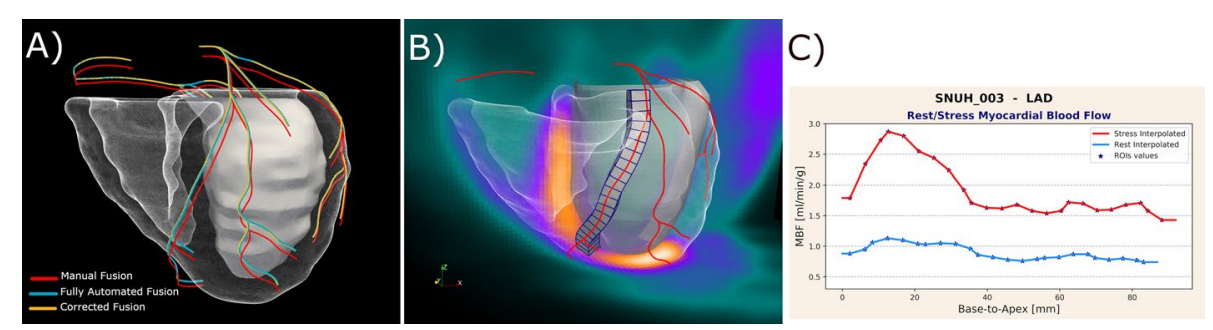

Fig. 2 A 3D display of CCTA-derived coronaries centerlines after fusion of dPET data with CCTA-derived anatomical masks obtained by manual delineation (red), with a fully automated segmentation algorithm (blue) and after manual revision of the automatically obtained masks (orange). B Extraction of the myocardium subtended to the LAD centerline and its discretization in ROls from which TACs will be derived and used in the model for MBF quantification. C Derived 2D plots of stress (red) and rest (blue) MBF ${ }^{v s}$ along the LAD length in $\mathrm{mm}$ from the base to the apex of the heart; markers * indicate the flow values obtained from the cubic ROIs; continuous curves obtained by interpolation. LAD left anterior descending artery, TACS time activity curves, $R O /$ region of interest

3 sets of post-fusion coronary centerlines showing limited differences in orientation and position, (B) the extraction of the myocardium subtended to a vessel and its discretization in ROIs, and $(C)$ the left anterior descending (LAD) r/s MBF ${ }_{M}^{v s}$ presented as a twodimensional (2D) graph of flow values along the vessel length (in $\mathrm{mm}$, from the base to the apex of the heart). The actual flow values obtained from the cubic ROIs are indicated by the markers.

For the current investigation, $\mathrm{r} / \mathrm{s} \mathrm{MBF}^{\mathrm{vs}}$ profiles were extracted for one vessel per vascular territory for each analyzed case: $\mathrm{LAD}$, one major vessel from the lateral wall (indicated as LCX), and the right posterior descending artery (rPDA). Figure 3A shows the anterior and posterior views of the heart with the vessel-specific modeling to probe three vessels for one of the analyzed cases. Figure 3B, C illustrates the differences introduced by the fusion of FA and $C$ models with respect to the reference standard $(M)$ in terms of LAD vessel-specific ROI spatial location.

\section{Normal/abnormal classification of $\mathrm{MBF}^{\mathrm{vs}}$ curves}

The $\mathrm{MBF}^{\mathrm{vs}}$ methodology was previously applied to define ranges of $\mathrm{r} / \mathrm{s} \mathrm{MBF}^{\mathrm{vs}}$ along the major vessels supplying the LV based on a cohort of patients $(n=15)$ with low risk of CAD who underwent ${ }^{13} \mathrm{NH}_{3}$ PET and CCTA (Piccinelli et al. 2020). Since the final goal of this approach is to determine whether indexes based on MBF become abnormal at specific locations along a vessel, thus indicating the presence of flow-limiting lesions that require revascularization, these low-risk ranges were used to prospectively stratify $\mathrm{MBF}^{\mathrm{vs}}$ into normal (within the range) versus abnormal (below the range). In the context of this investigation, the low-risk ranges were used to determine whether the three sets of $\mathrm{r} / \mathrm{s} \mathrm{MBF}^{\mathrm{vs}}$ curves were classified in the same way and/or to what extent the automated and the corrected curves differed from the ones obtained when the manually delineated anatomy was used for image registration. Figure 4 displays 2D plots of the low-risk ranges and $\mathrm{r} / \mathrm{s} \mathrm{MBF}_{\mathrm{M}, \mathrm{FA}, \mathrm{C}}^{\mathrm{vs}}$ for the three vessels considered for one of the analyzed cases.

To quantify the discrepancies in the normal/abnormal classification between $\mathrm{MBF}_{\mathrm{FA}}^{\mathrm{vs}}$ and $\mathrm{MBF}_{\mathrm{C}}^{\mathrm{vs}}$ compared to $\mathrm{MBF}_{\mathrm{M}}^{\mathrm{vs}}$ curves, the Dice coefficient (Dice 1945) (DC) was calculated. DC measures spatial overlapping commonly used to rate the performance of 

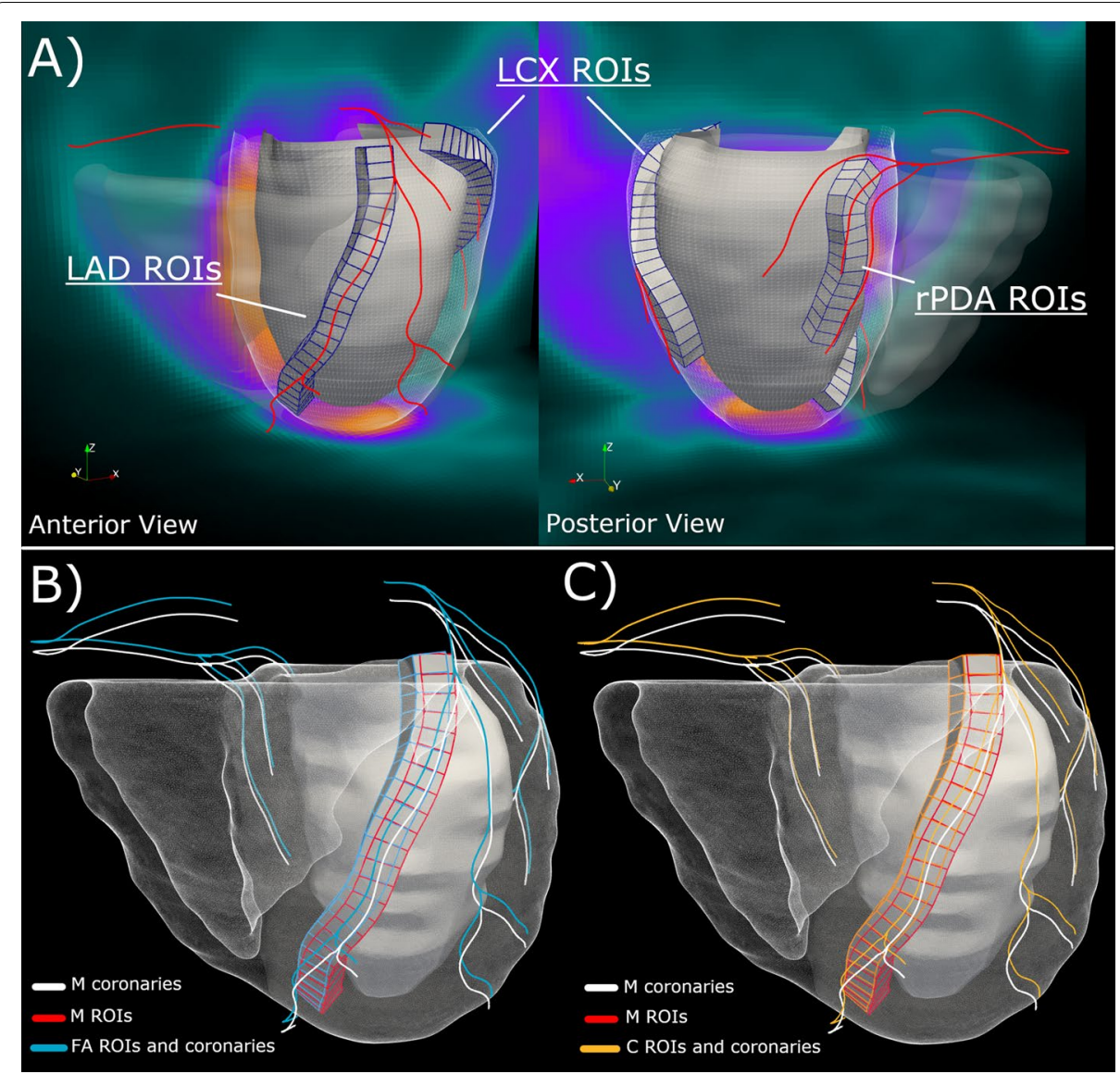

Fig. 3 A Anterior and posterior views of the heart 3D anatomy showing the extraction of the vessel-specific myocardium subtended to the LAD, LCX and rPDA and their discretization in ROIs for IPET sampling and TAC extraction. B Comparison between the manual LAD vessel-specific ROls (red) and the fully automated ROls (blue) with their corresponding centerlines. $\mathbf{C}$ same as in B comparing manual (red) and corrected (orange) LAD ROIs. M manual, FA fully automated. C corrected. RO/ region of interest, $L A D$ left anterior descending artery, LCX left circumflex artery, rPDA right posterior descending artery, TACs time activity curves

segmentation algorithms with values between 0 (no overlapping) to 1 (complete overlapping). Adapted to our analysis $\mathrm{DC}_{\mathrm{FA}}$ was computed to quantify the number of flow values (i.e., ROI values) with matched classification between $\mathrm{MBF}_{\mathrm{FA}}^{\mathrm{vs}}$ and $\mathrm{MBF}_{\mathrm{M}}^{\mathrm{vs}}$ based on low-risk ranges. Analogously, $\mathrm{DC}_{\mathrm{C}}$ was derived from the comparison of flow values classification between $\mathrm{MBF}_{\mathrm{C}}^{\mathrm{vS}}$ and $\mathrm{MBF}_{\mathrm{M}}^{\mathrm{vS}}$. $\mathrm{DC}_{\mathrm{FA}}$ and $\mathrm{DC}_{\mathrm{C}}$ were computed globally by pooling all the ROI samples from all the vessels. A parallel analysis was conducted per vascular territory as image quantification performance may differ among vascular territories.

\section{Inter-user variability analysis}

To assess the impact of the user corrections of the anatomical masks on the fusion procedure and consequently on the $\mathrm{MBF}^{\mathrm{vs}}$ profiles, a second user was tasked with correcting the automatically extracted anatomy in a subset of the analyzed cases. The second user had a similar background on cardiac anatomy and was provided with the same guidelines on how to perform the corrections. The cases were selected to represent different degree of agreement between $\mathrm{MBF}_{\mathrm{C}}^{\mathrm{vs}}$ and $\mathrm{MBF}_{\mathrm{M}}^{\mathrm{vs}}$. The $\mathrm{DC}_{\mathrm{C}}$ obtained after the two 


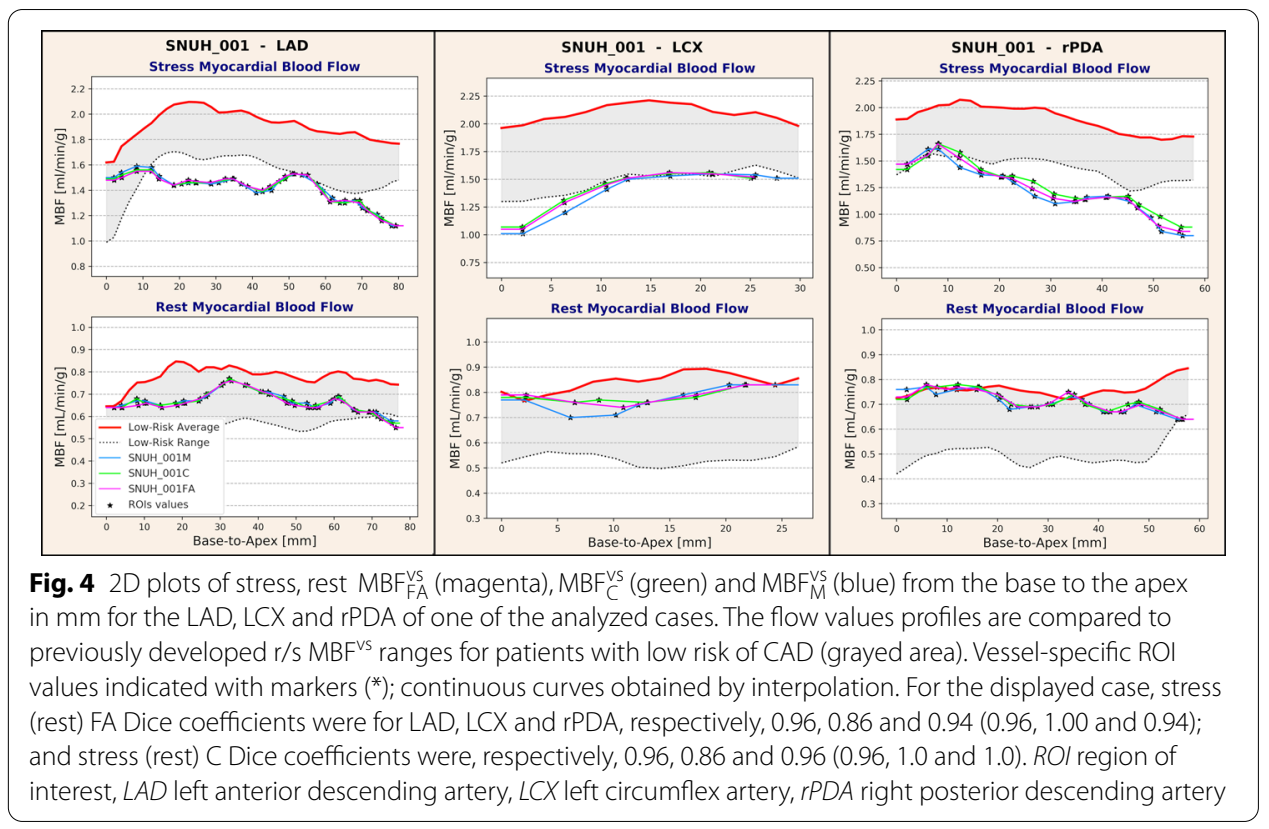

manual correction phases (indicated as $\mathrm{DC}_{\mathrm{C}}^{1}$ and $\mathrm{DC}_{\mathrm{C}}^{2}$ ) were calculated for a total of 12 vessels, three vessel per case.

\section{Statistical analysis}

Continuous variables are presented as mean $\pm S D$, while discrete variables are expressed as frequency distributions and percentages. The DC analysis was performed for all pooled vessels and separated by vascular territory and presented as mean values for each group. The presence of statistically significant differences in classification agreements between segmentation modes was tested by way of a Student's $t$ test, with $p<0.05$ as level of significance. The Student's $t$ test was also used to determine whether the two manual corrections of the anatomical masks provided significantly different classifications with respect to the normal/abnormal ranges.

\section{Results}

\section{Anatomical masks creation}

Forty-three consecutive patients collected from the imaging database of the DEMYSTIFY study were analyzed and processed for this investigation. Basic demographic characteristics and clinical data relevant for this investigation are summarized in Table 1 . The cohort contained a mix of patients with no physiologically significant $\mathrm{CAD}$ and patients with various degree of disease (as determined by invasive FFR index detailed in Table 1) to assess agreement in flow values in a variety of clinical scenarios. For each case M, FA and C anatomical masks (representing LV, RV and EPI) were defined from CCTA images (Fig. 1). The manual segmentation of the myocardium was time-intensive, ranging $1.5-2 \mathrm{~h}$ work per case. The fully automated segmentation was accomplished on average in $15 \mathrm{~s}$ per image, which confirms the clinical feasibility of the technique (Piccinelli et al. 2021). FA masks corrections 
Table 1 Demographic and clinical data for patients' population

\begin{tabular}{|c|c|c|c|}
\hline Patient demographic & All & $M$ & $\mathrm{~F}$ \\
\hline$n$ & 43 & 37 & 6 \\
\hline Age & 64 & 64 & 67 \\
\hline CAD risk factor & $n(\%)$ & & \\
\hline Hypertension & $31(72 \%)$ & & \\
\hline Diabetes & $13(30 \%)$ & & \\
\hline Hyperlipidemia & $38(88 \%)$ & & \\
\hline Tobacco use & $24(56 \%)$ & & \\
\hline Early CAD family history & $4(9 \%)$ & & \\
\hline Prior stents & $25(58 \%)$ & & \\
\hline Prior Ml & $4(9 \%)$ & & \\
\hline CABG & $0(0 \%)$ & & \\
\hline Heart failure & $0(0 \%)$ & & \\
\hline Vessels examined & FFR $<0.8$ & $F F R \geq 0.8$ & No FFR \\
\hline \multicolumn{4}{|l|}{$n=124$} \\
\hline LAD & 14 & 26 & 2 \\
\hline LCX & 4 & 20 & 18 \\
\hline rPDA & 3 & 24 & 13 \\
\hline
\end{tabular}

required a more variable time, depending on the quality of the automated segmentation results, as detailed below. Three centerlines following LAD, LCX and rPDA were manually delineated, with processing time that ranged 2-5 min per case. The three autonomous $\mathrm{r} / \mathrm{s}$ image registration procedures were performed, the coronary centerlines accordingly fused to the dPET data and the $\mathrm{MBF}^{\mathrm{vs}}$ extracted. With the exclusion of the anatomical information extraction (myocardium and centerlines), the successive processing was fully automated and ranged 2-3 min per vessel.

\section{PET/CCTA fusion}

Rest/stress image fusion was carried out for all cases with three sets of anatomical masks. In $37 \%$ of the 43 cases, no border corrections were necessary to complete the fusion, while editing was required for the remaining cases. As for their extent, the corrections were as follows: 1-border correction for 14/43 (33\%), 2-border correction for 16/43 (37\%) and 3-border correction for $7 / 43$ (16\%). The most common corrections were on the biventricular border (65\%), followed by the RV (53\%) and the LV profiles (35\%). The most common issue related to the FA segmentations was the inadequate coverage at the apex affecting only few slices of the total myocardial coverage; minimal adjustments were required for these cases which were achieved in 2-3 min on average. About $50 \%$ of the cases required such brief interventions which fell within the provided guidelines for manual corrections of the FA masks to create $\mathrm{C}$ masks. Situations with overlapping epicardium and endocardium (commonly present at the septum) required the simultaneous modification of two profiles, hence approximately twice the correction time. 


\section{MBFVS calculation and accuracy}

LAD, LCX and rPDA MBF $\mathrm{M}, \mathrm{FA}, \mathrm{C}$ were calculated for all cases that could be directly fused: 43 cases with $M$ masks, 43 cases with $C$ masks and 16 cases with intact FA masks. In three cases, no vessel suitable for analysis could be found in the LCX territory, and in 2 cases, the rPDA could not be identified from the CCTA images. A total number of 124 vessels were analyzed after fusion with the $M$ and $C$ masks yielding 2225 stress and 2122 rest flow values. Forty-seven vessels were analyzed after fusion with the FA masks producing 840 stress and 825 rest flow samples. Global and vessel-based DC were computed, and results are reported in Table 2. Figure 4 shows an example of good agreements between $\mathrm{MBF}_{\mathrm{FA}, \mathrm{C}}^{\mathrm{vs}}$ and $\mathrm{MBF}_{\mathrm{M}}^{\mathrm{vs}}$, and Fig. 5 displays cases with increasing DC values, from the worst case $(\mathrm{DC}=0.42)$ to instances of improved accuracy. 3D models and coronary centerlines are also displayed to assess the corresponding fusion results.

When analyzed on a case-by-case basis after fusion with $C$ masks, $63 \%$ and $85 \%$ of the vessels had perfectly matched $\mathrm{MBF}_{\mathrm{C}}^{\mathrm{vs}}$ values (i.e., $\mathrm{DC}=1$ ), respectively, for stress and rest, while $80 \%$ and $94 \%$ had stress and rest $\mathrm{DC}_{\mathrm{C}} \geq 0.9$, respectively. When FA masks were used, $62 \%$ and $72 \%$ of the vessels had perfectly matched stress and rest $\mathrm{MBF}_{\mathrm{FA}}^{\mathrm{vs}}$, while $83 \%$ and $94 \%$ had stress and rest $\mathrm{DC}_{\mathrm{FA}} \geq 0.9$. No statistical differences were found in the normal/abnormal classifications between manual and corrected or manual and fully automated, both globally and per vessel. When the 16 cases fused with native FA masks and $C$ masks were analyzed, no statistical differences could be found in the respective classification results.

\section{Inter-user variability analysis}

The 4 cases displayed in Fig. 5, which exhibited a wide range of agreements between the different $\mathrm{MBF}^{\mathrm{vs}}$ profiles and included the case with the worst performance in terms of fusion accuracy and consequently of $\mathrm{MBF}^{\mathrm{vs}}$ assessment, were used for the inter-user variability analysis. After manual corrections from the two users, $\mathrm{MBF}^{\mathrm{vs}}$ profiles were extracted along the LAD, LCX and rPDA of each case, which accounted for 223 stress and 218 rest flow values. Global $\mathrm{DC}_{\mathrm{C}}^{1}$ and $\mathrm{DC}_{\mathrm{C}}^{2}$ were, respectively, 0.86 and 0.85 at stress and 0.97 and 0.99 at rest. When vessels were separated by vascular territory $\mathrm{DC}_{\mathrm{C}}^{1}$ and $\mathrm{DC}_{\mathrm{C}}^{2}$ were for the LAD territory, respectively, 0.83 and 0.82 at stress, 0.97 and 1.00 at rest; for the LCX territory 0.89 and 0.84 at stress, 0.93 and 0.95 at rest; and for the RCA

Table 2 Agreement between $M \mathrm{MFF}_{\mathrm{FA}}^{\mathrm{Vs}}$ and $\mathrm{MBF}_{\mathrm{C}}^{\mathrm{Vs}}$ compared to $\mathrm{MBF}_{\mathrm{M}}^{\mathrm{Vs}}$ measured by way of the Dice coefficient

\begin{tabular}{|c|c|c|c|c|}
\hline & \multicolumn{2}{|c|}{ Manual versus fully automated masks } & \multicolumn{2}{|c|}{$\begin{array}{l}\text { Manual versus corrected } \\
\text { masks }\end{array}$} \\
\hline & Stress MBF & Rest MBF & Stress MBF & Rest MBF \\
\hline N samples & 840 & 825 & 2225 & 2122 \\
\hline Global Dice coefficient & 0.95 & 0.97 & 0.94 & 0.98 \\
\hline LAD Dice coefficient & 0.96 & 0.96 & 0.95 & 0.98 \\
\hline LCX Dice coefficient & 0.94 & 0.96 & 0.95 & 0.99 \\
\hline rPDA Dice coefficient & 0.95 & 0.98 & 0.93 & 0.98 \\
\hline
\end{tabular}

No statistically significant differences were found between global versus per territory assessments, between rest and stress MBF quantifications or due to the anatomical masks used to perform IPET/CCTA fusion 


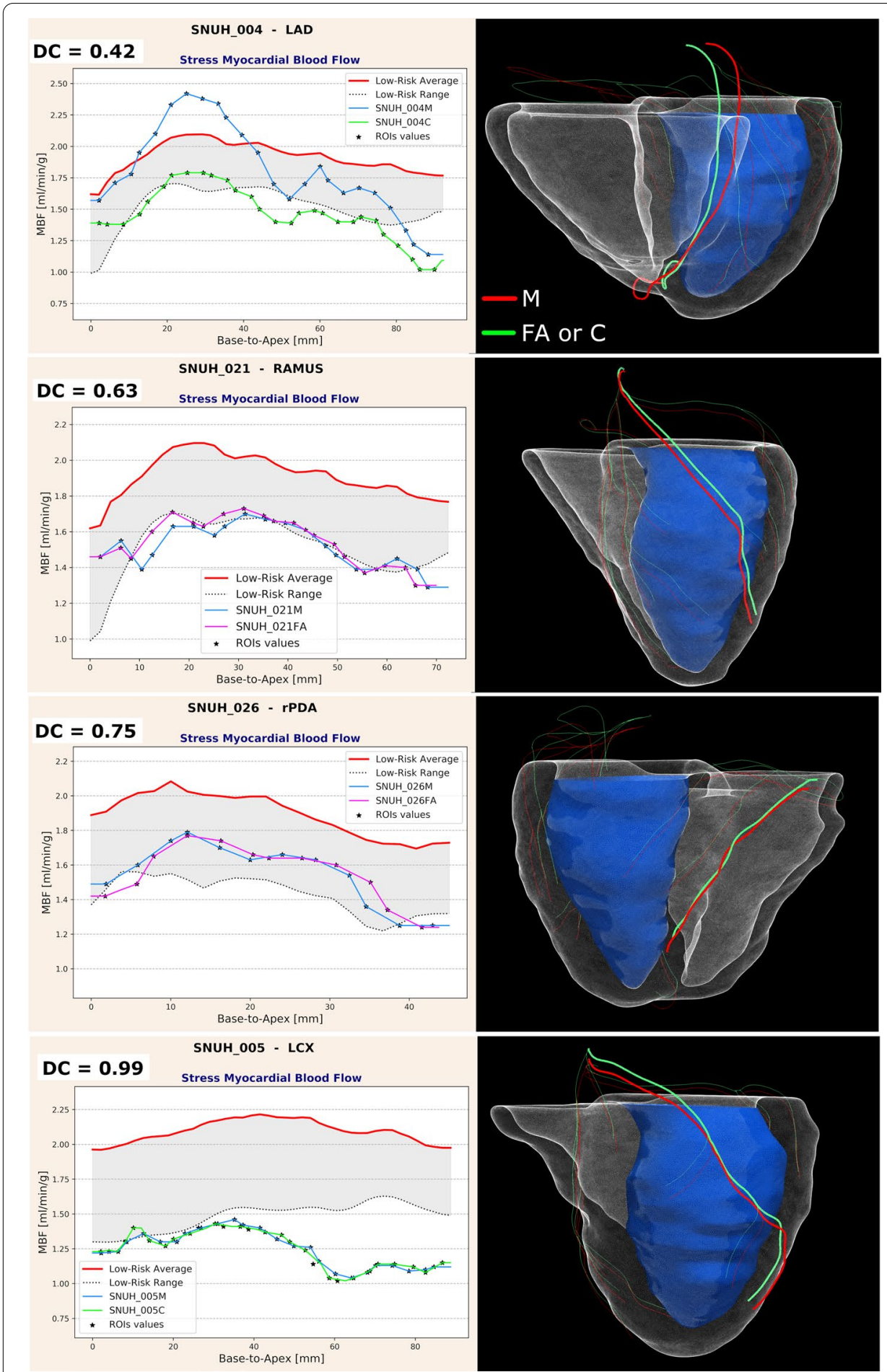

Fig. 5 Dice coefficient (DC) values for a series of analyzed vessels selected to show increased DC accuracy of $\mathrm{MBF}_{\mathrm{FA}}^{\mathrm{Vs}}$ or $\mathrm{MBF}_{C}^{\mathrm{Vs}}$ when compared to manual $\mathrm{MBF}_{\mathrm{M}}^{\mathrm{Vs}}$. Figure displays $2 \mathrm{D} \mathrm{MBF}$ profiles and correspondent $3 \mathrm{D}$ anatomies; thicker centerlines represent the analyzed vessel: Red centerlines are obtained from fusion with manual (M) anatomical masks and green centerlines from fusion with either fully automated (FA) or corrected (C) masks 
territory 0.87 and 0.94 at stress, 1.00 and 1.00 at rest. No statistically significant differences were found in the classification of individual vessels.

\section{Discussion}

In previous publications (Piccinelli et al. 2020; AlBadri et al. 2020), we presented a novel methodology to quantify MBF along vessel-specific 3D trajectories by fusing CCTA images to dPET. This multimodality image approach requires the efficient extraction of the heart anatomy from the CCTA acquisitions, namely blood pools and biventricular epicardium. In this work, we evaluated the ability of two sets of anatomical masks to guide efficiently and accurately image fusion and $\mathrm{MBF}^{\mathrm{vs}}$ extraction and permit its classification in normal versus abnormal values: the fully automated masks obtained with our previously developed algorithms for automated CCTA image segmentation (Dahiya et al. 2019; Piccinelli et al. 2021) and the corrected masks obtained after manual editing of the automated results by an expert user. The normal/abnormal classification of flow values was performed by comparing the calculated profiles to normality ranges previously developed from a cohort of subjects with low risk of CAD (Piccinelli et al. 2020). Our results indicate that both the automated (FA) and semi-automated (C) masks allow a highly accurate extraction of $\mathrm{MBF}^{\mathrm{vs}}$ along vessel trajectory that matched the values obtained when standard of reference manual segmentations were used (Table 2). The processing time from original images to $\mathrm{MBF}^{\mathrm{vs}}$ displays for the complete fully automated procedure was on average 5-7 min, compatible with the application of the methodology in a clinical environment. We determined that, although a majority of the fully automated borders required manual correction before $\mathrm{APET} / \mathrm{CCTA}$ image fusion, this editing process was brief (2-3 min) allowing a $100 \%$ success rate of extracting $\mathrm{MBF}^{\mathrm{vs}}$. Importantly, these $\mathrm{MBF}^{\mathrm{vs}}$ were shown to accurately categorize normal/abnormal flow along the major vessel path for both rest and stress flows. Moreover, for those cases that were processed with intact FA masks and $C$ masks, no significant differences were found in the normal/abnormal classification of $\mathrm{MBF}_{\mathrm{FA}}^{\mathrm{vs}}$ and $\mathrm{MBF}_{\mathrm{C}}^{\mathrm{vs}}$, substantiating our argument that our algorithms for automated segmentation of CCTA can correctly guide multimodality image fusion, notwithstanding the need for further improvements. Finally, by means of our small inter-user variability analysis, we showed that classification of flow values in normal/abnormal is unlikely to depend upon the user's limited and guided manual corrections of the anatomical masks.

The use of Dice coefficient to quantify the agreement between the different sets of MBF curves base to apex has also proved reliable. DC has commonly been used as an index of spatial overlapping specifically designed to grade the performance of different segmentation/classification methods. Since our interest is in establishing whether MBF becomes abnormal at specific locations along a vessel 3D trajectory, our proposed methodology can still be described as a classification technique, but with a different tolerance to pixel-level errors in the CCTA segmentations. Determining such a tolerance was the rationale for this work, and our results indicate that even if M, FA and C masks may exhibit differences, the PET coarser image resolution and the discretization of the myocardium subtended to the vessel of interest into ROIs shield MBF values from high variations and consequently major errors in flow assessment. Our implementation of the DC index was also relatively strict in rating the agreement between the different sets of 
$\mathrm{MBF}^{\mathrm{vs}}$ curves. The classification was overtly wrong only for a few vessels (3 vessels from 2 cases) and was characterized by $\mathrm{DC}<0.5$. But as depicted in the series of $2 \mathrm{D}$ plots in Fig. 5, even cases with MBF profiles that closely follows the standard of reference ones, the computed $\mathrm{DC}$ falls into a low-value range (0.6-0.75). Since our global $\mathrm{DC}_{\mathrm{FA}, \mathrm{C}}$ values are consistently $\geq 0.93$, these considerations further strengthen our conclusions on the technical robustness of the approach.

Gould et al. (Gould et al. 2000) introduced the concept of measuring relative tracer uptake base to apex on different LV quadrants and evidenced progressive abnormalities in uptake when considering different patients' populations from normal subjects to patients with diffuse disease, to patients with overt perfusion defects. Other groups (Valenta et al. 2014, 2017; Bom et al. 2019) have suggested measuring relative perfusion or absolute blood flow "longitudinally" with mixed results. With our approach we aim at overcoming the limitations related to the subdivision of the myocardium in standard territories by closely following 3D coronary paths irrespective of segments location and keeping the myocardial tissue probed to measure flow to a minimum in order to identify significant lesions.

Multimodal image approaches are powerful image-based diagnostic strategies. Yet numerous complicating factors have delayed the development of software tools that implement not just the visualization of synchronized images, but also a comprehensive and synergistic image quantification with extraction of indexes characterizing the anatomy and the function of the organ or the system under study. This work originated in our need to test the technical feasibility and quantify the robustness of our multimodal approach-provided the current state of development of our image processing toolsbut it also lays the foundations for the clinical validation of our $\mathrm{MBF}^{\mathrm{vs}}$ methodology and its potential in identifying flow-limiting lesions and guide revascularization.

\section{Limitations}

While we consider the results presented in this work very promising from the point of view of the clinical feasibility of our proposed method for $\mathrm{MBF}^{\mathrm{vs}}$ quantification, we recognize the need for additional steps toward its full automation. Further developments of our image segmentation algorithms are currently ongoing with a strong emphasis on the mitigation of the issues requiring manual corrections (i.e., incorrect myocardial thickness at the apex and portions of the septum). One additional limitation also related to the anatomy extraction is that vessel centerlines are currently manually delineated. The required processing is usually simple and quick to complete and as such, has not been the focus of our developments in automation. Nevertheless, numerous techniques exist for semi-automated retrieval of coronary centerlines to further make the methodology accessible and applicable (Li and Yezzi 2007) and will be investigated in future works.

\section{Conclusions}

Fully automated and manually corrected myocardial CCTA segmentation provide anatomical masks for vessel-specific myocardial blood flow measurements using dynamic PET/CCTA image fusion which are not significantly different in flow accuracy and within clinically acceptable processing times compared to fully manually segmented CCTA myocardial masks. 


\section{Abbreviations}

CAD: Coronary artery disease; PET: Positron emission tomography; CCTA: Coronary computed tomographic angiography; MBF: Myocardial blood flow; DC: Dice similarity coefficient; LV: Left ventricle; RV: Right ventricle; EPI: Epicardium.

\section{Authors' contributions}

All included authors have contributed to the design of the study (MP, EVG, HSB, BKK, AY, CDC, JN), the collection of the data (MP, DH, JCP, SGC, JML), the completion of the research (MP, RF, ND, DM, CDC, JN), and the writing and revision of the manuscript (all). All co-authors have reviewed and approved the current version of the manuscript.

\section{Funding}

Research reported in this publication was supported in part by NIH Grant R01 HL143350-03 from the National Heart, Lung and Blood Institute (PI EV Garcia). The content is the solely responsibility of the authors and does not necessarily represent the official views of $\mathrm{NIH}$.

\section{Availability of data and materials}

The datasets generated and/or analyzed in the current study are not publicly available but may be available from the corresponding author on reasonable request. The imaging data are currently being collected as part of the funded $\mathrm{NIH}$ R01 grant. Original imaging data and results will be made publicly available at the end of grant.

\section{Declarations}

Ethics approval and consent to participate

The study was conducted after local Institutional Review Board (IRB) committees' approval, and consent forms were obtained from all subjects.

\section{Consent for publication}

Not applicable.

\section{Competing interests}

Some of the authors (RF, CDC, EVG) receive royalties from the sale of the Emory Cardiac Toolbox and have equity positions in Syntermed, Inc. The terms of these arrangements have been reviewed and approved by Emory University in accordance with its conflicts of interest policies. The remaining authors did not report any conflicts of interest.

\section{Author details}

${ }^{1}$ Department of Radiology and Imaging Sciences, Emory University School of Medicine, 1364 Clifton Rd, NE, Atlanta, GA 30322, USA. ${ }^{2}$ Department of Electrical Engineering, Georgia Institute of Technology, Atlanta, GA, USA. ${ }^{3}$ Department of Internal Medicine and Cardiovascular Center, Seoul National University Hospital, Seoul, Korea. ${ }^{4}$ Department of Nuclear Medicine, Seoul National University Hospital, Seoul, Korea. ${ }^{5}$ Department of Nuclear Medicine, Chonnam National University, Gwangju, Korea. ${ }^{6}$ Samsung Medical Center, Heart Vascular Stroke Institute, Seoul, Korea.

Received: 20 October 2021 Accepted: 4 December 2021

Published online: 15 February 2022

\section{References}

AlBadri A, Piccinelli M, Cho SG et al (2020) Rationale and design of the quantification of myocardial blood flow using dynamic PET/CTA-fused imagery (DEMYSTIFY) to determine physiological significance of specific coronary lesions. J Nucl Cardiol 27:1030-1039. https://doi.org/10.1007/s12350-020-02052-0 (Epub 2020 Feb 5)

Bom MJ, Driessen RS, Raijmakers PG et al (2019) Diagnostic value of longitudinal flow gradient for the presence of haemodynamically significant coronary artery disease. Eur Heart J Cardiovasc Imaging 20:21-30

Dahiya N, Yezzi A, Piccinelli M et al (2019) Integrated 3D anatomical model for automatic myocardial segmentation in cardiac CT imagery. CMBBE Imaging vis. https://doi.org/10.1080/21681163.2019.1583607

de Bruyne B, Sarma J (2008) Fractional flow reserve: a review. Heart 94:949-959

de Bruyne B, Pijls NHJ, Kalesan B et al (2012) Fractional flow reserve-guided PCI versus medical therapy in stable coronary disease. N Engl J Med 367:991-1001

Dice LR (1945) Measures of the amount of ecologic association between species. Ecology 26:297-302

Faber TL, Santana CA, Piccinelli M et al (2011) Automatic alignment of myocardial perfusion images with contrastenhanced cardiac computer tomography. IEEE Trans Nucl Sci 58:2296-2302

Gould KL, Nakagawa Y, Nakagawa K et al (2000) Frequency and clinical implications of fluid dynamically significant diffuse coronary artery disease manifest as graded, longitudinal, base-to-apex myocardial perfusion abnormalities by noninvasive positron emission tomography. Circulation 101:1931-1939

Hutchins GD, Schwaiger M, Rosenspire KC et al (1990) Noninvasive quantification of regional blood flow in the human heart using N-13 ammonia and dynamic positron emission tomographic imaging. J Am Coll Cardiol 15:1032-1042

Lee JM, Kim CH, Koo BK et al (2016) Integrated myocardial perfusion imaging diagnostics improve detection of functionally significant coronary artery stenosis by $13 \mathrm{~N}$-ammonia positron emission tomography. Circ Cardiovasc Imaging 9:e004768. https://doi.org/10.1161/CIRCIMAGING.116.004768

Li H, Yezzi A (2007) Vessels as 4D curves: global minimal 4D path to extract 3D tubular surfaces and centerlines. IEEE Trans Med Imaging 26:1213-1223 
Nye JA, Piccinelli M, Hwang D et al (2021) Dynamic cardiac PET motion correction using 3D normalized gradient fields in patients and phantom simulations. Med Phys. https://doi.org/10.1002/mp.15059

Piccinelli M, Santana CA, Sirineni GKR et al (2018) Diagnostic performance of the quantification of myocardium at risk from MPI SPECT/CTA 2G fusion for detecting obstructive coronary disease: a multicenter trial. J Nucl Cardiol 25:1378-1386

Piccinelli M, Cho SG, Garcia EV et al (2020) Vessel-specific quantification of absolute myocardial blood flow, myocardial flow reserve and relative flow reserve by means of fused dynamic 13NH3 PET and CCTA: Ranges in a low-risk population and abnormality criteria. J Nucl Cardiol 27:1756-1760 (Epub Oct 2018)

Piccinelli M, Dahiya N, Folks RD et al (2021) Validation of automated biventricular myocardial segmentation from coronary computed tomographic angiography for multimodality image fusion. MedRxiv. https://doi.org/10.1101/2021. 03.08.21252480

Schindler TH, Quercioli A, Valenta I et al (2014) Quantitative assessment of myocardial blood flow_clinical and research applications. Semin Nucl Med 44:274-293

The Vascular Modelling Toolkit (VMTK). www.vmtk.org. Accessed 12 July 2021.

Valenta I, Quercioli A, Schindler TH (2014) Diagnostic value of PET measured longitudinal flow gradient in the identification of coronary artery disease. JACC Cardiovasc Imaging 7:387-396

Valenta I, Antoniou A, Marashdeh W et al (2017) PET-measured longitudinal flow gradient correlates with invasive fractional flow reserve in CAD patients. Eur Heart J Cardiovasc Imaging 18:538-548

\section{Publisher's Note}

Springer Nature remains neutral with regard to jurisdictional claims in published maps and institutional affiliations.

\section{Submit your manuscript to a SpringerOpen ${ }^{\odot}$ journal and benefit from:}

- Convenient online submission

- Rigorous peer review

- Open access: articles freely available online

- High visibility within the field

- Retaining the copyright to your article

Submit your next manuscript at $\mathbf{s p r i n g e r o p e n . c o m ~}$ 